\title{
Model Predictive Control for Power Optimization in a Hydrostatic Wind Turbine
}

\author{
Feng Wang and Kim A. Stelson \\ Center for Compact and Efficiency Fluid Power \\ Department of Mechanical Engineering, University of Minnesota \\ Minneapolis, MN 55455 USA \\ E-mail: wang2148@umn.edu, kstelson@umn.edu
}

\begin{abstract}
Model predictive control (MPC) is applied to a mid-sized hydrostatic (HST) wind turbine for maximizing power capture in this paper. This study focuses on the torque control in region 2, which tracks the desired rotor speed so that the turbine can operate at the optimum tip-speed ratio (TSR) for maximum power. Preliminary study shows that the widely used $K \omega^{2}$ control law has a good control performance in steady-state wind conditions. However due to wind turbulence, the turbine operates at tip-speed ratios far away from the optimal point. This deviation is not only due to the large rotor inertia, but also due to the characteristics of the $K \omega^{2}$ control. An MPC controller is proposed to track the desired rotor speed by using the future prediction of wind speed. To consider the potential advantage, the MPC controller is applied to a $50 \mathrm{~kW}$ HST wind turbine. A wind speed step change is selected as a basic test of transient response. The control performance of the MPC is evaluated and compared with the $K \omega^{2}$ control law. Results show that the MPC controller in a smaller wind speed step change shows a faster response than $K \omega^{2}$ control law, but a large overshoot is observed. In a larger wind speed change, the MPC controller loses control when the wind speed steps down. This indicates the MPC controller in this study has limited effective operation range since it uses a linearized plant model and the wind turbine is a highly nonlinear system. Future work includes the optimization of MPC controller parameters to reduce the overshoot during the wind speed change and the design of multiple MPC controllers for wide operation range.
\end{abstract}

Keywords: Mid-sized wind turbine, hydrostatic transmission, wind turbulence, power optimization, model predictive control

\section{Introduction}

Wind power is a plentiful, renewable source of energy, able to produce emission-free power in the kilowatt to megawatt range. Land-based or off-shore wind farms can connect several hundred wind turbines to the grid. However, these facilities require expensive power transmission lines and typically incur significant construction and maintenance costs. For areas with smaller power needs, such as farms or factories, a small wind facility is a cost-effective method of power generation. These mid-sized wind turbines often have a fixed rotor speed which reduces cost by eliminating the power converter. However, since the tip-speed ratio (TSR) cannot be optimized, fixed speed operation does not allow the rotor to capture the maximum energy as the wind speed varies. To capture wind energy more efficiently, a continuously variable transmission (CVT) is required.

A hydraulic CVT in the form of a hydrostatic transmission (HST) provides a competitive solution for a mid-sized wind turbine. With a hydrostatic transmission, the generator speed is decoupled from the rotor speed, making it possible to use a synchronous generator. The generator can run at synchronous speed at different wind speeds and rotor speeds, eliminating the use of a power converter. There are also some other advantages of using an HST in a wind turbine, such as more damping to reduce shock loading, flexible system configuration, low cost and high reliability [1].

There are four control regions in a wind turbine. In region 1 where the wind speed is below cut-in speed, the turbine is in stand-by mode. In region 2 where the wind speed is between the cut-in and the rated speed, the turbine is controlled so that it runs at the optimum TSR to achieve maximum power. In region 3 where the wind speed is above the rated but below the cut-out speed, the turbine output is limited to the rated power. In region 4 where the wind speed is above the cut-out speed, the turbine is shut down to avoid damage. To achieve maximum energy capture in region 2 , a widely used control strategy in the wind industry, referred to as the 
$K \omega^{2}$ control law, is used. The beauty of the $K \omega^{2}$ law is that it only needs rotor speed information and does not require the wind speed information. The control law can automatically bring the turbine to the optimum operation point by controlling the rotor reaction torque $[2,3]$.

Preliminary study shows that the $K \omega^{2}$ law gives good control performance in steady-state wind conditions [4]. However in the real world, wind speed varies both temporally and spatially. Wind turbulence in the form of wind gusts, happen unexpectedly. During wind turbulence, the turbine operates at tip-speed ratios far away from the optimum TSR even with the $K \omega^{2}$ law. This is not only due to the large inertia of the blade, but also due to the characteristics of the $K \omega^{2}$ law in which the rotor speed is regulated indirectly by controlling the rotor reaction torque. In a wind gust, the large inertia of the rotor prevents the rotor speed from changing fast enough to adapt to the increased wind speed. On the other hand, the rotor reaction torque increases slowly due to slowly increasing rotor speed according to $K \omega^{2}$ law, which tends to slow down the rotor angular acceleration.

Various control methods for wind turbines have been recently studied. A typical feedback controller adjusts the rotor angular speed to desired values based on the wind speed information. However, even if the feedback controller can use the wind speed information, the delay between the control action and the resulting response cannot be avoided since the inertia of the blade is fairly large. The use of the future wind information has been discussed to further improve the control performance in some studies. If the controller can preview the wind speed information, this would lead to significant improvements in the control performance.

In this paper we consider applying model predictive control to rotor speed control. An MPC controller is designed to track desired rotor speed by using estimates of future wind speed. In the design it is assumed that the MPC controller can use wind speed over some future time horizon from a few seconds to minutes. The future prediction of wind speed can be obtained using a statistical model. Also, the use of LIght Detection And Ranging (LIDAR) technology to measure the wind speed is possible. MPC has been considered for control of conventional gearbox turbines for power optimization and load reduction $[5,6,7,8,9]$. This is the first study to consider MPC control of a hydrostatic wind turbine.

\section{Hydrostatic wind turbine control - baseline}

\subsection{Control hierarchy}

A modern gearbox turbine has several levels of control systems. In the high level, a supervisory controller monitors the wind speed and determines when the wind speed is sufficient to start up the turbine and when the turbine must be shut down for safety due to high wind speeds. The middle level control mainly includes the torque control and the blade pitch control. Torque control, controlled through the power electronics in gearbox turbines, determines how much torque is extracted from the rotor shaft. The extracted torque opposes the rotor aerodynamic torque provided by the wind and thus indirectly regulates the rotor speed. The low control level includes the generator, power electronics and pitch actuator controllers, which operate much faster than the middle-level control. The control hierarchy of the gearbox wind turbine is shown in Figure 1.

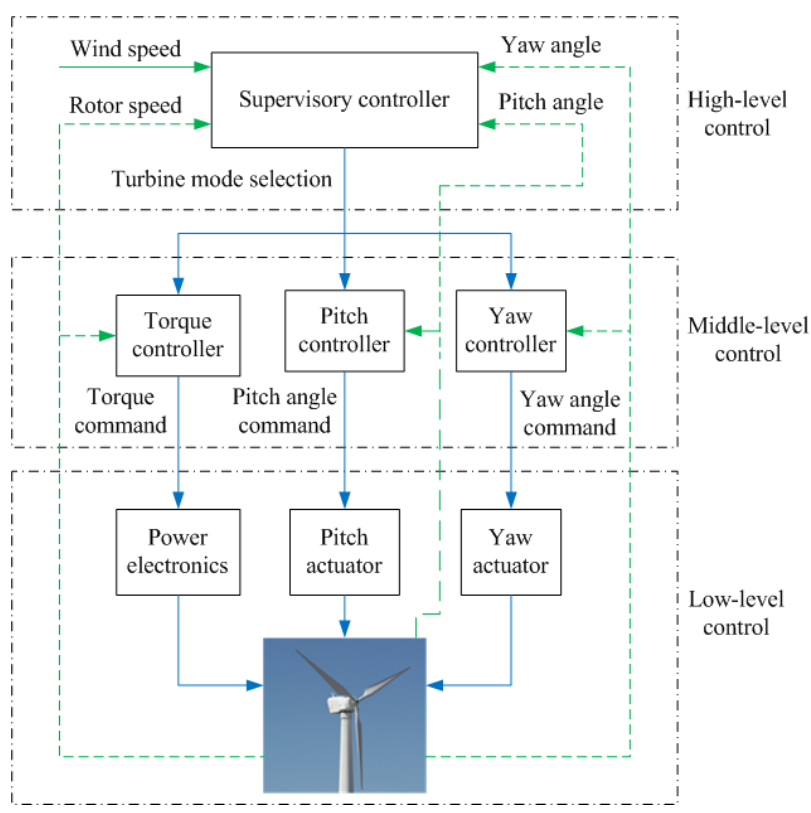

Figure 1 Control hierarchy of gearbox wind turbine

For variable-speed turbines operating in region 2 , the control objective is to maximize energy capture by operating the turbine at the optimum TSR. This is achieved by the torque controller. The pitch controller is not active in this region. The focus of this study is torque control in region 2 . The $K \omega^{2}$ control law is used as the baseline in this study.

\subsection{Baseline $K \omega^{2}$ control law}

The power coefficient $C_{p}$ is a function of the tip-speed ratio $\lambda$ and the blade pitch angle $\beta$, which is represented by a surface. The optimum TSR to reach the maximum power coefficient shifts with the blade pitch angle. In region 2 where the blade is at a fine pitch angle, the optimum TSR is fixed. The TSR, $\lambda$, is defined as:

$$
\lambda=\frac{\omega R}{v}
$$

where $\omega$ is the rotor angular speed, $R$ is the radius of the blade and $v$ is the wind speed.

In $K \omega^{2}$ control, the control torque (rotor reaction torque), $\tau_{c}$, is given by:

$$
\tau_{c}=K \omega^{2}
$$

where $K$ is the control gain given by:

$$
K=\frac{1}{2} \rho A R^{3} \frac{\mathrm{C}_{\text {pmax }}}{\lambda_{*}^{3}}
$$


where $\rho$ is the air density, $A$ is the blade swept area and $\lambda_{*}$ is the optimum TSR at which the maximum power coefficient $C_{\text {pmax }}$ occurs.

The beauty of the $K \omega^{2}$ law is that it only needs rotor speed information and does not require wind speed information. The control law can automatically bring the turbine to the optimum operation point since the optimum operation parameters, $\lambda_{*}$ and $C_{\text {pmax }}$, are included in the control gain, $K$. The $K \omega^{2}$ law can track the optimum TSR well if accurate turbine parameters, $\lambda_{*}$ and $C_{p \max }$, are given.

\subsection{Hydrostatic wind turbine control}

The schematic diagram of a hydrostatic wind turbine is shown in Figure 2. The low-speed rotor shaft drives a fixed displacement pump and the high-speed generator is driven by a variable displacement hydraulic motor.

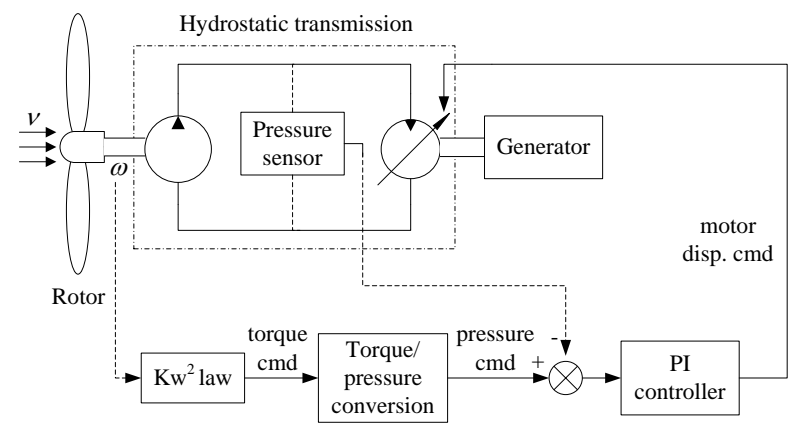

Figure 2 Schematic diagram of a hydrostatic wind turbine

The control of an HST turbine is similar to a gearbox turbine except for the torque control in the middle-level. In an HST turbine, instead of controlling the generator torque through power electronics, the rotor reaction torque (pump torque) is determined by the line pressure which is controlled by varying the motor displacement. By using a PI controller to track the desired line pressure, the desired control torque can be achieved.

The relationship between the control torque, $\tau_{c}$, and the line pressure, $p_{c}$, is:

$$
p_{c}=\frac{\tau_{c} \eta_{p m}}{D_{p}}
$$

where $D_{p}$ is the pump displacement and $\eta_{p m}$ is the pump mechanical efficiency.

A simulation model of the hydrostatic wind turbine is built in Simulink. The model is a physical equation based dynamic simulation model which simulates both the quasi-static and the dynamic conditions. The input and output causality of each component is verified by the bond graph method. The detailed system modeling can be found in our previous work [10].

\section{Model predictive control for HST wind turbine}

\subsection{Overview}

The fundamental idea of MPC is illustrated in Figure 3. At current time $k$, the MPC controller predicts the future behavior of a plant over a prediction horizon by using a plant model and computes a control input sequence which minimizes a certain performance cost function by solving an optimization problem. Only the first element of the control input sequence is applied to the plant. At next time step $k+1$, based on the new measurements, the MPC controller predicts the future behavior over the shifted prediction horizon and computes a new control input sequence. Similarly, only the first element of the newly computed control input sequence is applied to the plant. The above procedure is repeated as time shifts. During solving the optimization problem, MPC also takes the input and output constraints into considerations.

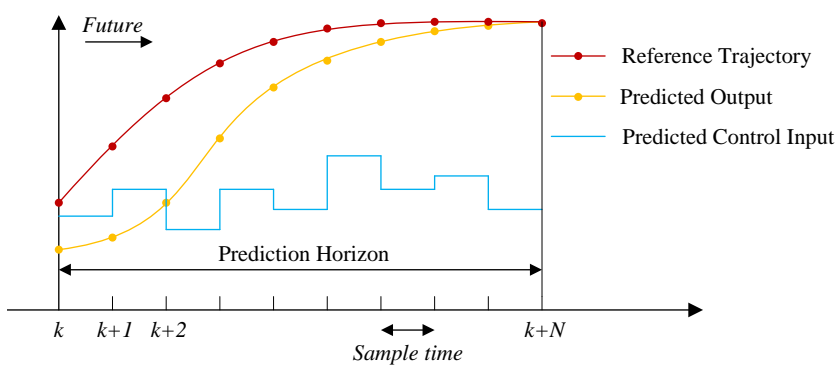

Figure 3 Scheme of model predictive control

While applying MPC to an HST wind turbine, the reference signal of the MPC controller is the desired rotor speed based on the future wind speed information. The system output, rotor speed, is feedback to the MPC controller. The wind speed is input to the MPC controller as a measured disturbance. The MPC controller uses an internal plant model and optimizer to calculate the control input to the plant, which is in the current case the pump torque command. The MPC controller in the hydrostatic turbine is shown schematically in Figure 4.

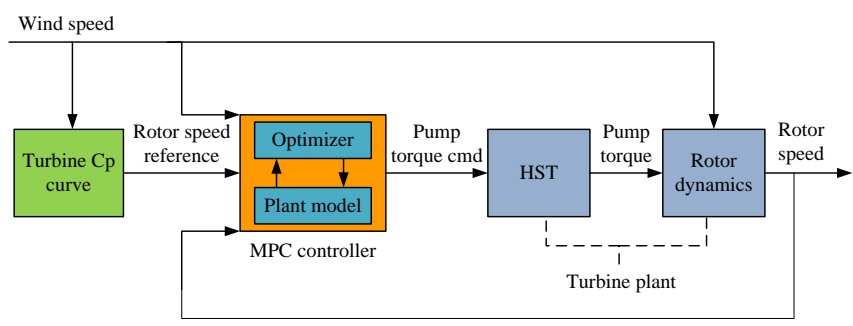

Figure 4 MPC controller in hydrostatic wind turbine

The objective function over the prediction horizon $N$ is:

$$
J=\sum_{i=0}^{N-1}\left[Q\left(\omega_{r \mid k+i}-\omega_{k+i}\right)^{2}+S\left(\delta \tau_{c \mid k+i}\right)^{2}\right]
$$

where $Q$ and $S$ are weighting factors.

The optimization minimizes the rotor speed tracking error $\omega_{r \mid k+i}-\omega_{k+i}$ and the pump torque command variation $\delta \tau_{c \mid k+i}$ over the prediction horizon while satisfying the input and output constraints. In an HST 
wind turbine, the maximum rotor speed is limited by the blade design. The maximum pump torque is determined by the pump displacement and maximum line pressure. The second term of the cost function is to penalize the pump torque variation since there are physical limitations on how fast the pump torque can change.

The MPC controller uses a linearized plant model to calculate control inputs. Therefore the MPC controller is an approximation of the system behavior since there is discrepancy between the linearized model and the actual plant. If the plant is highly nonlinear within the operation range, the MPC controller designed for the specific operation point may not have satisfactory control performance throughout the operation range. The wind turbine system is such a system where the rotor torque increases nonlinearly with wind speed and rotor speed.

\section{Simulation study}

\subsection{Simulation parameters}

The case studied in this paper is a mid-sized wind turbine. The rotor aerodynamic model is from AOC 15/50, which is a $50 \mathrm{~kW}$ wind turbine manufactured by Atlantic Orient Corporation. The rotor aerodynamic model of AOC $15 / 50$ is generated using FAST code. FAST is a multibody wind turbine dynamics code developed by NREL [11]. In the simulation model, the rotor aerodynamic torque is generated by a 2D loop-up table taking the wind speed and rotor speed as input indexes.

Instead of designing an MPC controller for the entire operation range, the current study considers one operation point and the plant model is linearized at this point. In the simulation, the selected operation point is at the wind speed of $6 \mathrm{~m} / \mathrm{s}$. To compare the MPC controller with the $K \omega^{2}$ control law, a step change on the wind speed is considered. The main simulation parameters are shown in Table 1.

Table 1 Main simulation parameters

\begin{tabular}{lll}
\hline \hline Property & Value & Unit \\
\hline Blade radius & 7.5 & $\mathrm{~m}$ \\
Rotor swept area & 176.7 & $\mathrm{~m}^{2}$ \\
Rated rotor speed & 88 & $\mathrm{rpm}$ \\
Optimum TSR & 6.1 & - \\
Maximum Cp & 0.45 & - \\
Air density & 1.225 & $\mathrm{~kg} / \mathrm{m}^{3}$ \\
Pump displacement & 1570 & $\mathrm{cc} / \mathrm{rev}$ \\
Motor displacement & 71 & $\mathrm{cc} / \mathrm{rev}$ \\
Pipeline internal diameter & 32 & $\mathrm{~mm}$ \\
Pipeline length & 2 & $\mathrm{~m}$ \\
Wind speed at operation point & 6 & $\mathrm{~m} / \mathrm{s}$ \\
Rotor speed setpoint at $6 \mathrm{~m} / \mathrm{s}$ & 4.88 & $\mathrm{rad} / \mathrm{s}$ \\
Pump torque at 6 m/s & 2156 & $\mathrm{Nm}$ \\
Control interval & 0.02 & $\mathrm{~s}$ \\
Prediction horizon & 200 & - \\
Control horizon & 50 & - \\
Pump torque rate weight, $S$ & 0.1 & - \\
Rotor speed weight, $Q$ & 20 & - \\
\hline \hline
\end{tabular}

\subsection{Simulation results}

The wind turbine system is linearized at the wind speed of $6 \mathrm{~m} / \mathrm{s}$ using the Matlab linearization toolbox. This linearized model is then used in the MPC controller to solve the optimization problem. Figure 5 shows the wind speed changes and the corresponding rotor speed changes using the $K \omega^{2}$ and MPC controllers. The wind speed steps up from 6 to $7 \mathrm{~m} / \mathrm{s}$ at $60 \mathrm{~s}$ and steps down from 7 to $6 \mathrm{~m} / \mathrm{s}$ at $120 \mathrm{~s}$.
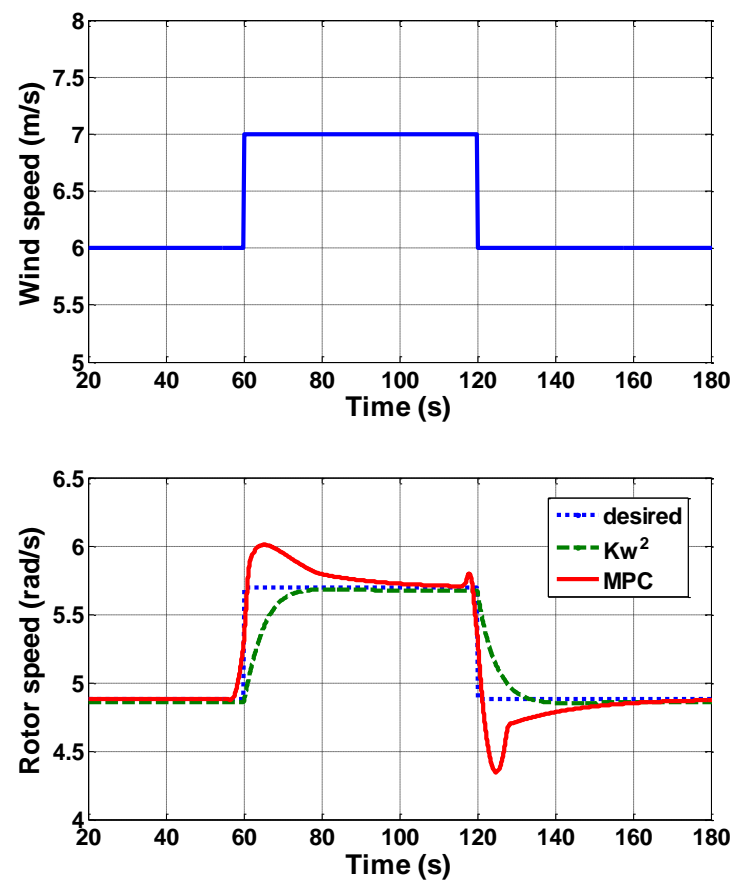

Figure 5 Wind speed change from 6 to $7 \mathrm{~m} / \mathrm{s}$ and the corresponding rotor speed changes using the $K \omega^{2}$ and MPC controllers

The desired rotor speed is calculated using the wind speed information. It is shown that the rotor speed using $K \omega^{2}$ controller slowly approaches the desired point without overshoot. The rotor speed using MPC controller changes before the wind speed change because it uses future wind speed information. The MPC controller shows a shorter rise time than the $K \omega^{2}$ controller. However a large overshoot is observed and the settling time is longer. Since the $1 \mathrm{~m} / \mathrm{s}$ wind speed change is within the linear range of the model, the same response will be seen for smaller steps.

To show the response of the controller in the nonlinear range, a $2 \mathrm{~m} / \mathrm{s}$ wind speed step change is simulated. Figure 6 shows the response using the $K \omega^{2}$ and MPC controllers. The wind speed steps up from 6 to $8 \mathrm{~m} / \mathrm{s}$ at $60 \mathrm{~s}$ and steps down from 8 to $6 \mathrm{~m} / \mathrm{s}$ at $120 \mathrm{~s}$. When the wind speed steps up, the results are similar to previous simulation results. However, the MPC controller loses control when the wind speed steps down from 8 to $6 \mathrm{~m} / \mathrm{s}$. This indicates the MPC controller in the wind turbine system has a limited effective operation range since the linearized plant model at the wind speed of $6 \mathrm{~m} / \mathrm{s}$ may not work well when the wind speed is too far away from 
that wind speed. To solve this problem, multiple MPC controllers at different operation points are required and the control may switch among different MPC controllers according to the wind speed.

Comparing the simulation results in Figure 5 and Figure 6 , it is also shown that the MPC controller has an asymmetric behavior when the wind speed steps up and down. In Figure 5, the overshoot when the wind speed steps down is larger than the overshoot when the wind speed steps up. While in Figure 6, the situation when the wind speed steps down is even worse.
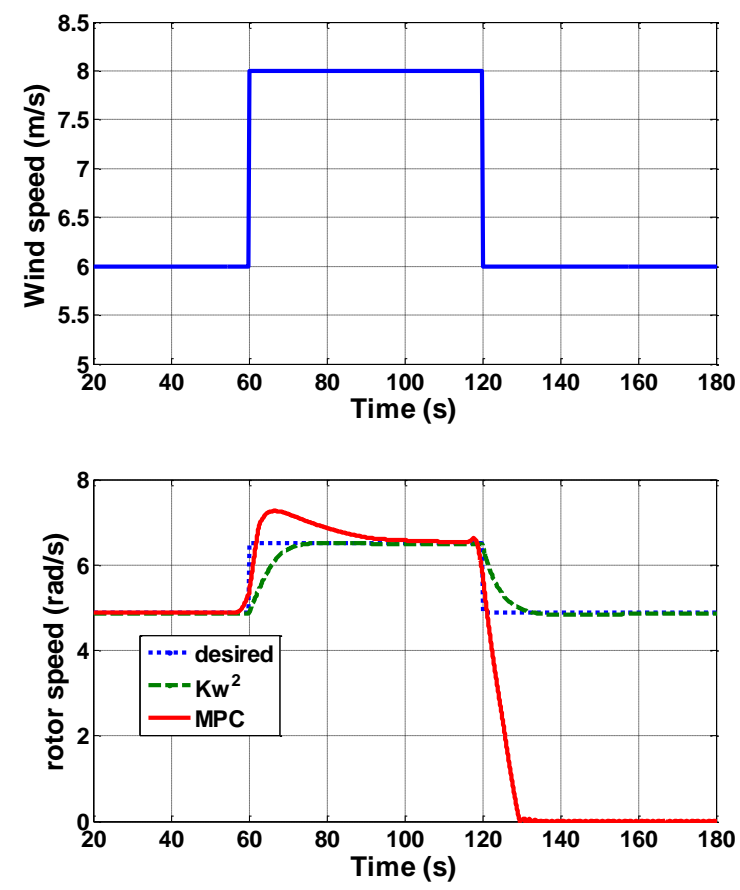

Figure 6 Wind speed change from 6 to $8 \mathrm{~m} / \mathrm{s}$ and the corresponding rotor speed changes using the $K \omega^{2}$ and MPC controllers

\section{Conclusions and discussions}

In this paper a model predictive control is proposed for maximizing power capture in a mid-sized hydrostatic wind turbine. The study focuses on the torque control in region 2, which tracks the desired rotor speed so that the turbine can operate at the optimal tip-speed ratio for maximum power. Preliminary study shows that the well developed $K \omega^{2}$ law has a good control performance in steady-state wind conditions. However during wind turbulence, the turbine operates at tip-speed ratios far away from the optimum TSR. This is not only due to the large rotor inertia, but also due to the characteristics of the $K \omega^{2}$ law itself.

An MPC controller is proposed to track the desired rotor speed by using the future prediction of wind speed. The controller uses a linearized plant model to solve an optimization problem that minimizes the rotor speed tracking error and the control input variation over the prediction horizon while satisfying the input and output constraints. To consider the potential advantage, the
MPC controller is applied to a $50 \mathrm{~kW}$ HST wind turbine. The plant model is linearized at the wind speed of $6 \mathrm{~m} / \mathrm{s}$ and then used in the MPC controller.

The control performance of the MPC is evaluated and compared with the $K \omega^{2}$ control law in the simulation. Two wind speed step changes ( 1 and $2 \mathrm{~m} / \mathrm{s}$ ) are studied. Results show that the MPC controller in a smaller wind speed change has a faster response than $K \omega^{2}$ control law, however a large overshoot is observed. In a larger wind speed change, the MPC controller loses control when the wind speed steps down. This indicates the MPC controller in this study has a limited effective operation range since the linearized plant model at the wind speed of $6 \mathrm{~m} / \mathrm{s}$ may not work well when the wind speed is far away from that wind speed.

Based on the results of this study, there are still many improvements needed before applying the MPC controller to wind turbines. One future task is to optimize the MPC control parameters to reduce the overshoot during the wind speed change. Another task is to design multiple MPC controllers and switch among different MPC controllers at different wind speeds.

\section{Acknowledgments}

This research was supported by the Center for Compact and Efficient Fluid Power, a National Science Foundation Engineering Research Center funded under cooperative agreement number EEC-0540834.

\section{References}

[1] Thul, B., Dutta, R., Stelson, K. A., Hydrostatic transmission for mid-sized wind turbines. $52^{\text {nd }}$ National Conference on Fluid Power, Las Vegas, USA, 2011.

[2] Johnson, K. E., Pao, L. Y., Balas, M. J., Fingersh, L. J., Control of variable-speed wind turbines: standard and adaptive techniques for maximizing energy capture. IEEE Control Systems Magazine, vol.26, no.3, pp.70-81, 2006.

[3] Pao, L. Y., Johnson, K. E., A tutorial on the dynamics and control of wind turbines and wind farms. American Control Conference, pp. 2076-2089, St. Louis, Missouri, USA, 2009.

[4] Wang, F., Trietch, B., Stelson, K. A., Mid-sized wind turbine with hydro-mechanical transmission demonstrates improved energy production. The 8th International Conference on Fluid Power Transmission and Control (ICFP 2013), Hangzhou, China, 2013.

[5] Henriksen, L. C., Model predictive control of a wind turbine, master thesis, Technical University of Denmark, 2007.

[6] Soltani, M., Wisniewski, R., Brath, P. and Boyd, S., Load reduction of wind turbines using receding horizon control, Proceedings IEEE MultiConference on Systems and Control, pp. 852-857, 2011. 
[7] Zhongzhou, Y., Yaoyu, L., and Seem, J. E., Model predictive control for wind turbine load reduction under wake meandering of upstream wind turbines, American Control Conference, 2012.

[8] Mostafa, S., Malik, O. P., and Westwick, D. T., Multiple model predictive control for wind turbines with doubly fed induction generators. IEEE Transactions on Sustainable Energy, vol.2, no.3, 2011.

[9] Khalid, M., Savkin, A.V., A model predictive control approach to the problem of wind power smoothing with controlled battery storage, Renewable Energy, vol.35, pp.1520-1526, 2010.

[10] Dutta, R., Wang, F., Bohlmann, B., Stelson, K. A., Analysis of short-term energy storage for mid-size hydrostatic wind turbine. ASME Dynamic Systems and Control Conference, Fort Lauderdale, FL, USA, 2012.

[11] NWTC design codes (FAST by Jason Jonkman). http://wind.nrel.gov/designcodes/simulators/fast/ 